\title{
Gestión de residuos químicos en la Universidad Estatal a Distancia: abordajes desde la Regencia Química
}

\author{
Eric Montero Miranda ${ }^{1}$, Eduard Rios Badilla²
}

1. Universidad Estatal a Distancia, Escuela de Ciencias Exactas y Naturales, Programa de Laboratorio, Regencia Química, B Escalante, San José, Costa Rica; emonterom@uned.ac.cr

2. Universidad Estatal a Distancia, Escuela de Ciencias Exactas y Naturales, Programa de Laboratorio, Regencia Química, B ${ }^{\circ}$ Escalante, San José, Costa Rica; eriosb@uned.ac.cr

3.

Recibido: 01 de Abril de $2020 \quad$ Aceptado: 25 de Junio de 2020

\begin{abstract}
RESUMEN
Este trabajo describe las estrategias empleadas por la Regencia Química del Programa de Laboratorio (PROLAB) de la Universidad Estatal a Distancia (UNED) para la gestión de los residuos químicos peligrosos generados, producto de la actividad académica de los laboratorios de ciencias, así como de los diversos centros y proyectos de investigación adscritos a la universidad. Como parte de las acciones de esta unidad se identifican, segregan, almacenan y se gestiona el tratamiento de alrededor de $208 \mathrm{~L}$ y $25 \mathrm{~kg}$ anuales de residuos de sustancias químicas clasificados en ácidos inorgánicos, disolventes orgánicos, halogenados, alcalinos, aceites, metales, siendo en su mayoría los residuos líquidos de metales pesados. Además, se neutralizan cerca de $60 \mathrm{~L}$ de sustancias ácidas y básicas por año y se envían mil envases contaminados a un gestor autorizado para su tratamiento. El año de mayor gestión de residuos fue el 2019 y finalmente se lograron disponer los residuos equivalentes a $75 \mathrm{~kg}$ de vidrio contaminado con mercurio para el periodo 2017-2019. Estos esfuerzos han reafirmado el compromiso de la institución con la salud del ambiente y de sus colaboradores.
\end{abstract}

Palabras claves: residuo químico, gestión química, gestión de residuos, sustancias peligrosas, tratamiento.

\section{ABSTRACT}

Chemical Residues management at Universidad Estatal a Distancia: chemical stewarship approach

This work describes the strategies used by the Chemical Management Office within the Laboratory Program (PROLAB) of the State University of Distance Education (UNED) for the management of hazardous chemical waste generated, product of the academic activity of scientific courses, as well as the various research centers and projects attached to the university. As part of the responsibilities of this unit, the treatment of around $208 \mathrm{~L}$ and $75 \mathrm{~kg}$ per year of chemical waste takes places, being classified in six categories in order to be identified, segregated, stored and managed. The principal waste identified was the heavy metal liquid waste. In addition, about $60 \mathrm{~L}$ of acidic and basic substances are neutralized, and a thousand contaminated containers are sent to an authorized agent for treatment. Additionally, most of the management activity was developed during 2019 and the equivalent to $75 \mathrm{~kg}$ of glassware waste contaminated with mercury was properly disposed between 2017 and 2019. This paper concludes with some future perspectives for the appropriate chemical waste management. These results serve as evidence of the institution`s commitment with its people and environmental health.

Key words: chemical waste, chemical management, waste management, hazardous substances, treatment. 


\section{Introducción}

La gestión de residuos químicos involucra la reducción de la cantidad de sustancias peligrosas generadas, el tratamiento para reducir su toxicidad y la aplicación de procesos de control que además reduzcan o eliminen la exposición a dichos contaminantes (Vallero, 2019). Los residuos químicos son generados al final de un proceso, en donde se ha utilizado alguna sustancia química y su pureza ha sido modificada, por ejemplo, un análisis químico que requiera el uso de disolventes (Decreto 27000-MINAE, 1998).

Mantener un sistema que permita al ente productor separar los residuos desde su generación es de vital importancia para su almacenamiento y tratamiento posterior (FSE, 2010). Por otro lado, para lograr una adecuada gestión de residuos, es necesario definir jerarquías en las estrategias de gestión para tomar las decisiones más adecuadas para la disposición de los residuos que genere la empresa (Martínez, 2005). (Ver figura 1).

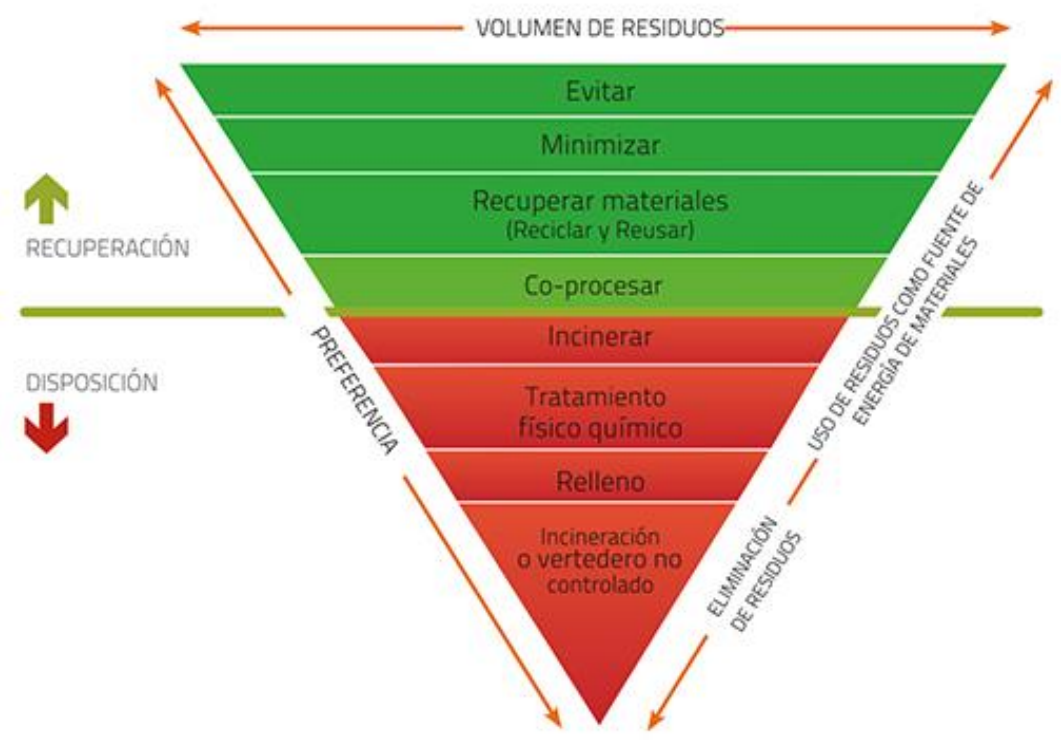

Figura 1. Estrategia jerarquizada para la gestión integral de los residuos (Geocycle, 2019)

También, es importante que en el lugar de almacenamiento los trabajadores porten los implementos de seguridad recomendados, así como las medidas de seguridad para su traslado (Poder Ejecutivo, 1998). El tipo de tratamiento que reciba el residuo dependerá de los potenciales efectos derivados del manejo de estos. En muchos casos, su tratamiento implica una inversión muy grande por los elevados costos y la complejidad de las sustancias (Mora, 2010). A pesar de que en el país los medios para el tratamiento son muy limitados, existen métodos de incineración como los que ofrece las empresas Holcim o CEMEX, además de métodos de inmovilización, como la estabilización o la solidificación (Vargas et al, 2006).

El Programa de Laboratorio de la Universidad Estatal a Distancia, es un programa que se encuentra adscrito a la Escuela de Ciencias Exactas y Naturales que fue creado en 1978 como parte de la necesidad que tenía la universidad de poseer una unidad que gestione las actividades desarrolladas. Inicialmente, en los cursos que incluyen asignaturas de laboratorio de ciencias de la ECEN (Acontecer, 2018). Actualmente, el Programa de Laboratorio se encarga de la logística de relacionada con la asesoría en proyectos de construcción, acondicionamiento y desarrollo de asignaturas de laboratorio, para las carreras que oferta la ECEN, además, brinda soporte a proyectos de extensión, investigación y a estudiantes en condición de tesis (PROLAB, 2019). La UNED ha experimentado un crecimiento anual de $2,9 \%$ en su matrícula y cerca del $20 \%$ de la población estudiantil debe de cursar al menos una materia de laboratorio en ciencias. Por otro lado, la infraestructura de la universidad ha ido en aumento, para el 2011 existían 10 laboratorios y para finales de 2020 se proyectan 20 laboratorios más y con esto se deberá gestionar mayor cantidad de residuos por parte del PROLAB (UNED, 2012).

En Costa Rica, la preocupación por las sustancias químicas utilizadas y los residuos generados en el país ha ido creciendo con los años, esto debido a la toma de conciencia de los entes generadores, que ha repercutido en las mejoras a nivel nacional, sobre el impacto que provoca la producción y/o el uso inadecuado de sustancias químicas peligrosas, sobre la salud y el ambiente (GNC, 2008). Para 1992 se organizó la Conferencia de las Naciones Unidas sobre el Medioambiente y Desarrollo en Río de Janeiro (Cumbre de Río), en donde se crea la "Agenda 21”. Este documento 
que tiene como objetivo generar un compromiso en los Estados miembros de las Naciones Unidas para realizar un desarrollo sostenible, en su capítulo 19, expone el tema relacionado con productos químicos bajo el tratado para la "Gestión Ecológicamente Racional de los Productos Químicos, incluida la Prevención del Tráfíco Internacional Ilícito de Productos Tóxicos y Peligrosos", la cual, los Estados debían cumplir para el 2000 (GNC, 2008). Costa Rica fue uno de los países que suscribió este acuerdo y desde entonces ha invertido esfuerzos en mejorar la gestión de sustancias peligrosas y sus residuos (GNC, 2008; Ministerio de Salud, 2011). Instituciones como el Centro Nacional de Producción más Limpia, el Ministerio de Salud, Ministerio de Ambiente y Energía y dentro de este último la Dirección de Gestión de Calidad Ambiental (DIGECA), han trabajado para desarrollar normativas que regulen la gestión de sustancias peligrosas y sus residuos.

Para 1998 se crean los decretos 2700-MINAE "Reglamento sobre las características y listado de los desechos peligrosos industriales" que incluye en sus anexos los códigos SIMARDE (Sistema para el Monitoreo de Aguas Residuales y Desechos) para la clasificación de residuos y familias de residuos y el decreto 27001-MINAE "Reglamento para el manejo de los desechos peligrosos industriales". En el 2010 se renuevan estos últimos a través de la Ley N8839 para la "Gestión Integral de Residuos". Para el 2010, a través del Ministerio de Salud, se establece el Política Nacional para la Gestión Integral de Residuos 2010-2021, que brinda una perspectiva preventiva y bajo una responsabilidad social compartida, con la idea de que estos adopten procesos sostenibles de producción y consumo, así como en el manejo seguro y ambientalmente adecuado de los residuos generados en diferentes ámbitos (Ministerio de Salud, 2011). Por otro lado, comienzan a conformarse empresas que poseen una certificación extendida por DIGECA para la gestión de residuos, brindando el servicio de recolección, almacenamiento y tratamiento de residuos para empresas que así lo deseen.

El contexto de la gestión de residuos generados en los laboratorios de la UNED ha mejorado desde la creación de la Regencia Química en el 2007, bajo la creación de protocolos como resultado de la implementación de la normativa costarricense. Los primeros esfuerzos aparecen en el 2017, como parte del crecimiento en los laboratorios de ciencias e investigación de la UNED, la apertura de nuevos cursos en sus diferentes sedes y el aumento en el consumo de sustancias químicas y con ello, de los residuos generados. Actualmente, la UNED cuenta con un sistema donde se clasifican los residuos peligrosos en seis categorías según el decreto 27000-MINAE. Además, se tienen protocolos para su segregación, etiquetado, disposición final y protocolos auxiliares que brindan medidas en caso de derrame y para su transporte. Los residuos recolectados y almacenados son tratados de forma cuatrimestral mediante una empresa autorizada y certificada por la DIGECA para la gestión de residuos peligrosos. También se ha trabajado en programas de capacitación del personal para la gestión adecuada de residuos.

\section{Metodología}

Para implementar un sistema de gestión de residuos adecuado para la UNED, se realizó un diagnóstico de las acciones que existían en la institución al momento de la implementación. Inicialmente, se realizó una revisión de los inventarios de sustancias químicas y de los manuales de los cursos de laboratorio impartidos por las diferentes carreras. Luego de extraer el tipo de sustancias empleadas en cada curso, se procedió a clasificar las sustancias químicas por sus peligrosidades, así se pudo crear un total de seis familias de residuos a los que además se les asignó un color para su fácil identificación. Se realizó un diseño para las etiquetas de acuerdo con lo recomendado por el Sistema Globalmente Armonizado de Clasificación y Etiquetado de Productos Químicos (SGA, Naciones Unidas), las bitácoras de recolección de residuos y las boletas de disposición final del residuo. Por último, se estableció la relación comercial para el tratamiento de las sustancias con un gestor autorizado por el Ministerio de Salud (Ministerio de Salud, 2019).

Los residuos son recolectados y segregados en cada laboratorio de ciencias de la UNED y luego son transportados para su posterior pretratamiento en el PROLAB, en el cual, se mide la temperatura y el $\mathrm{pH}$, se neutraliza una parte de los residuos y el resto es trasvasado a contenedores de mayor volumen y enviados a un posterior tratamiento con un gestor autorizado. Después de implementar todo el sistema de gestión, se realizaron las labores de capacitación del personal técnico y los profesores tutores de los cursos de química. Como parte de estas tareas, se desarrolló material didáctico que posteriormente ayudaría al personal docente y estudiantil a segregar los residuos generados en las actividades académicas.

\section{Resultados}

Como parte de la implementación de las metodologías se logró recolectar, segregar, almacenar y gestionar el tratamiento de $623,48 \mathrm{~L}$ de residuos líquidos y $75 \mathrm{~kg}$ de vidrio contaminado con mercurio (Hg) entre el 2017 y el 2019. El cuadro 1, exhibe las clasificaciones principales clasificaciones y las cantidades de residuos recolectados y gestionados entre el 2017 y el 2019. 
Cuadro 1. Principales residuos peligrosos generados en la UNED en el periodo 2017-2019

Tipo de residuo

Color de la etiqueta Cantidad (L)

\begin{tabular}{lcc}
\hline Ácido Inorgánicos y mezclas de ácidos mordientes* & Celeste & 118 \\
\hline Mezcla de disolventes orgánicos & Rojo & 130 \\
\hline Disolventes halogenados y mezclas de disolventes halogenados & Verde & 70 \\
\hline Disoluciones alcalinas* & Morado & 90 \\
\hline Aceites sin contenidos de bifenilos policlorados & & 10 \\
\hline Concentrados y semi-concentrados con sales metálicas & Amarillo & \\
\hline Termómetros de mercurio** & Negro & 200 \\
\hline Total & No aplica & 5,48 \\
\hline
\end{tabular}

* De esta categoría se neutralizaron 60 L, el resto se almacenó como residuos al encontrarse contaminado con otra sustancia.

** Se descarta esta sustancia en concordancia al Protocolo de Minamata, además se trataron 75 kg contaminados con mercurio proveniente de los termómetros.

Los residuos fueron recolectados en 11 sedes con un total de 17 laboratorios, para el 2020 se estima que el número de nuevos laboratorios sea de 9, los cuales se suman al sistema de gestión. Por otro lado, se espera la incorporación de 3 sedes que tendrían un laboratorio cada una para inicios del 2021. En la figura 2 se observa la distribución de tipos de residuos en los últimos tres años

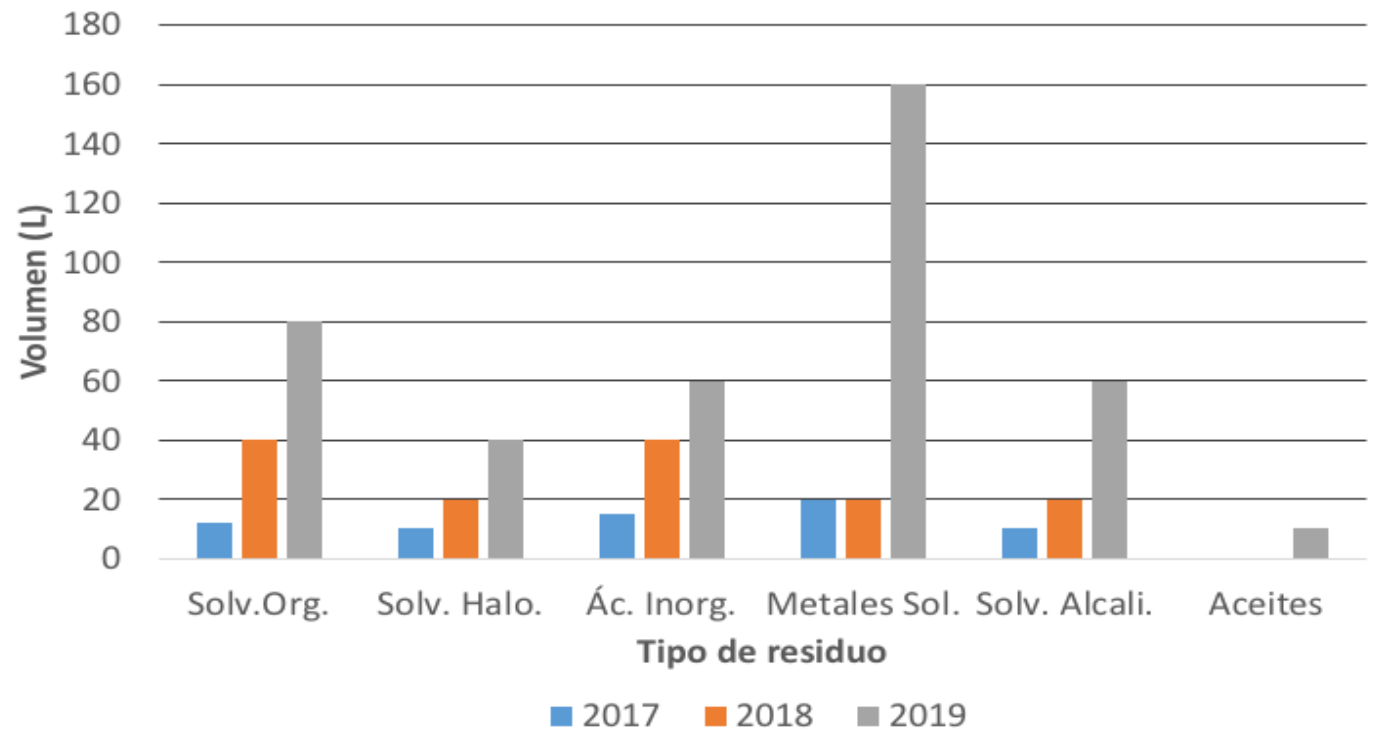

Figura 2. Distribución anual de residuos tratados en la UNED en el período 2017-2019.

El 2019 fue el año de mayor actividad en la gestión, con un $66 \%$ del tratamiento total de este período. También, los residuos de aceites se comienzan a tratar a partir del 2019, ya que estos no tenían una generación muy alta, sin embargo, a partir del 2020 se espera un aumento en la generación de este residuo, dado que comienza a operar el laboratorio de ingeniería industrial en la sede de Alajuela. Con los demás residuos, se estima que tengan un comportamiento similar al presentado en la Figura 2, en donde se tiene un aumento anual. 
Los residuos recolectados y enviados al PROLAB reportaron un valor promedio de temperatura de $26{ }^{\circ} \mathrm{C}$. Con respecto al $\mathrm{pH}$, este último fue ajustado con disoluciones de bicarbonato de sodio o ácido acético diluido, según sea el caso, para establecer valores entre 6,5 y 7,5, antes de llenar la boleta final de disposición final de cada residuo. En la Figura 3 se muestran las operaciones de neutralización y recolección de residuos para su posterior tratamiento mediante un gestor externo.

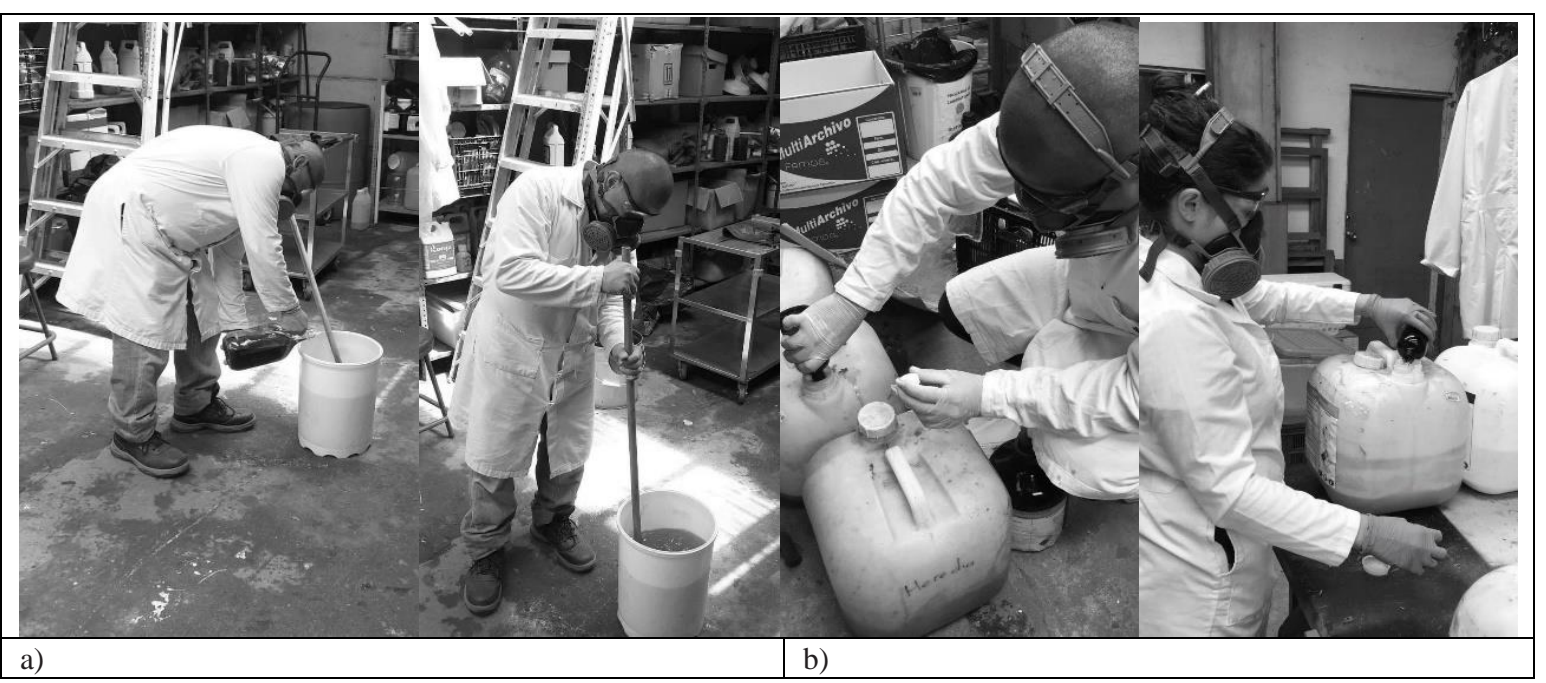

Figura 3. a) Proceso de neutralización ácido-base. b) Proceso de segregación de residuos en las instalaciones del PROLAB.

La fase de capacitación fue la última etapa del sistema, en la cual, se realizaron sesiones de trabajo con los técnicos de laboratorio y los tutores de las asignaciones de laboratorio. Por otro lado, se implementaron afiches explicativos (ver figura 4) y se generaron protocolos que apoyen la labor de gestión de residuos.

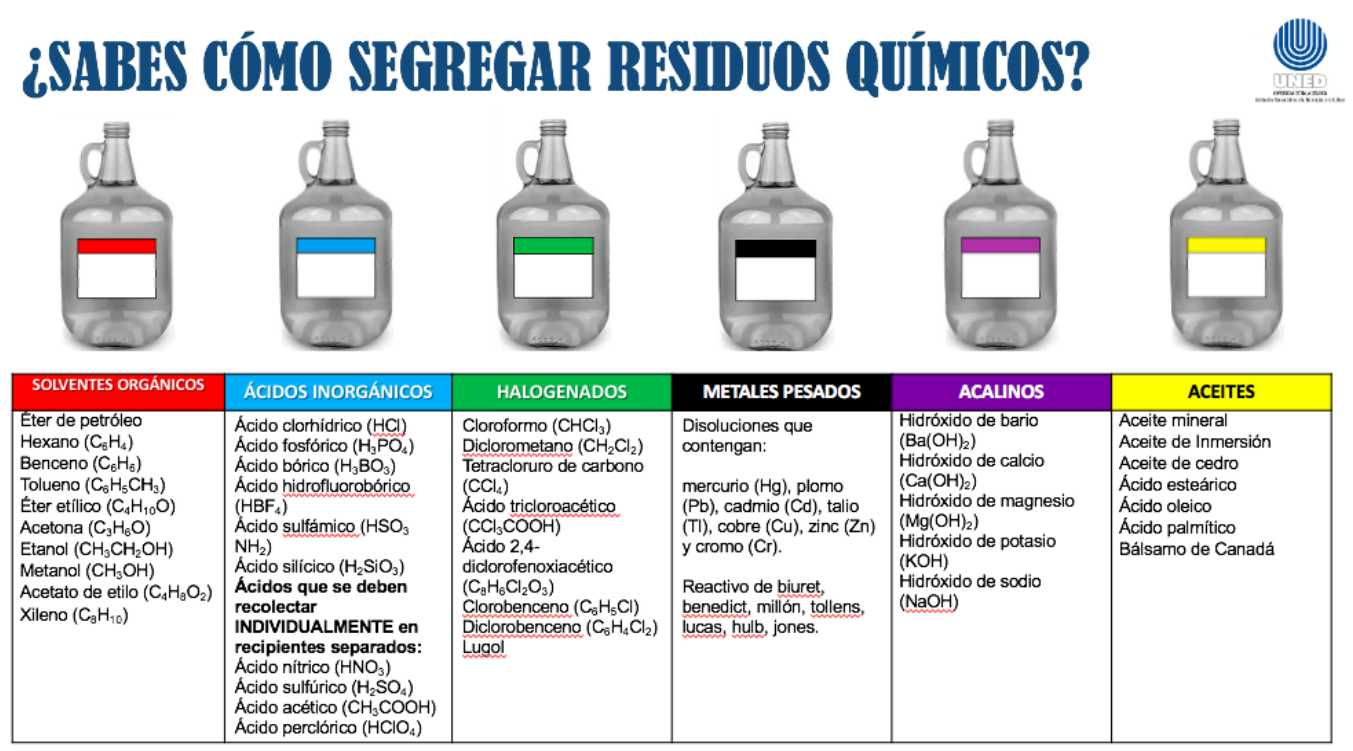

Figura 4. Afiche para la segregación correcta de residuos químicos generados en el laboratorio.

\section{Discusión}

La gestión de residuos constituye una práctica responsable y su importancia radica en los beneficios que esta representa para la institución y para el ambiente. Por otro lado, las estrategias desarrolladas no solo ayudan a la institución con el cumplimiento legislativo, sino también aportan herramientas que enriquecen los esfuerzos de programas como Bandera Azul Ecológica y el Programa de Gestión Ambiental Institucional (PGAI), ambos, de carácter obligatorio para las entidades públicas del país. 
Como se pudo observar en la Figura 2, existe un aumento en la generación de residuos producidos en le universidad, esto obedece al crecimiento que ha experimentado la institución como parte del reforzamiento y de la construcción de nuevos laboratorios de docencia y de investigación en los últimos tres años, dada la inversión del Banco Mundial. Se estima que, para los siguientes dos años, este incremento se mantenga, ya que, está pendiente la inauguración de tres laboratorios en Parrita, Upala y La Cruz. La institución requiere generar conciencia en los trabajadores, capacitarlos para reconocer los tipos de residuos y su correcto manejo, su segregación y disposición final. Asimismo, deben de realizar un plan de acción, contemplando las actividades que se realizan en cada área diariamente y determinando los factores de riesgo (CONAMA, 2005). Sería prudente en trabajos futuro incluir el análisis de riesgo por sede universitaria para generar un análisis del panorama nacional de la institución. Si bien, las estrategias implementadas en la UNED han enriquecido la gestión de residuos, es necesario implementar procedimientos para la mejora continua de las mismas, con el fin de perfeccionar cada vez más esta labor.

A pesar del corto tiempo, la unidad de Regencia Química y el PROLAB han podido implementar un proyecto con datos positivos, la mayoría de los residuos peligrosos generados por la UNED se han podido gestionar de una adecuada manera, sin embargo, es necesario expandir esta iniciativa más allá de los laboratorios de ciencias e investigación y platearlo como un proyecto institucional en el que se involucren a distintos actores y otros proyectos de acción social, docencia y extensión universitaria.

\section{Conclusiones}

El desarrollo de un sistema para la gestión de residuos peligrosos permite tomar acciones para la disminución de estos. Un factor que influye en la generación de residuos es la gestión de los productos químicos que lo generan, por lo que se deben implementar prácticas docentes que permitan sustituir, eliminar o reducir el uso de sustancias que puedan representar la generación de un residuo químico peligroso.

Dado que el sistema de gestión de residuos nace como una estrategia para cumplir requerimientos legislativos, es importante que se implemente bajo un análisis del ciclo de vida de los productos químicos, esto permite disminuir los impactos ambientales de la institución y establecería un punto de partida para futuras certificaciones en este campo.

Si bien la gestión de residuos químicos representa un gasto para la institución, los beneficios ambientales que se perciben son mayores, y permiten contribuir en la mejora continua y suma esfuerzos dentro de los programas ambientales de la institución. En este sentido, la gestión de residuos es un tema económico también, al evitar costos por multas o compensaciones ambientales.

A pesar de que la institución tiene poco tiempo trabajando con la gestión de residuos, la implementación de estas estrategias ha permitido recolectar y tratar más de $620 \mathrm{~L}$ de residuos y $75 \mathrm{~kg}$ de sólidos, lo que representa una disminución significativa de sustancias que pudieron en algún momento ser liberadas al ambiente y un compromiso por la gestión efectiva para la disminución de los riesgos asociados a los residuos químicos peligrosos.

\section{PERSPECTIVAS A FUTURO}

Recuperación de disolventes: Durante el proceso de identificación y clasificación de residuos fue posible reconocer el potencial que posee la unidad para recuperar un volumen considerado de disolventes orgánicos utilizados en las actividades de docencia e investigación, por lo que estas actividades se han definido dentro del plan de trabajo para el próximo periodo. La rectificación de disolventes genera beneficios económicos para la institución al comprar menor cantidad de los mismos, y además, ayuda a disminuir nuestra huella ecológica al minimizar el volumen de material orgánico desechado.

Escalamiento y sustitución: se podría pensar en el uso de experimentos a menor escala con el fin de disminuir la cantidad de residuos generados, así como evitar la dilución innecesaria durante la experimentación. Esto es algo que se tendrá que trabajar en conjunto con las cátedras docentes. Por último, algo que ya se ha hecho a través de los años, pero no deja de ser objeto de vigilancia continua del programa, es la sustitución de sustancias nocivas para el ambiente por aquellas que muestren con el tiempo ser más seguras, según la evidencia científica.

\section{Agradecimientos}

Agradecemos a la Mag. Ana Ligia Mora Garro, coordinadora del Programa de Laboratorio (PROLAB) de la Universidad Estatal a Distancia (UNED), por su apoyo y gestión para que este proyecto fuera una realidad en la institución, así como a la dirección de la Escuela de Ciencias Exactas y Naturales. También, a todo el personal técnico y profesional del PROLAB, que ha trabajado arduamente en mantener este proyecto en marcha. Al jefe de Servicios Generales de la UNED, quién gestionó el apoyo económico para el primer año de este proyecto. 


\section{Declaración de intereses}

Ética, conflicto de intereses y declaración de financiamiento: los autores declaran haber cumplido con todos los requisitos éticos y legales pertinentes, tanto durante el estudio como en el manuscrito; que no hay conflictos de interés de ningún tipo, y que todas las fuentes financieras se detallan plena y claramente en la sección de agradecimientos. Asimismo, están de acuerdo con la versión editada final del documento. El respectivo documento legal firmado se encuentra en los archivos de la revista.

Ethical, conflict of interest and financial statements: the author(s) declare that they have fully complied with all pertinent ethical and legal requirements, both during the study and in the production of the manuscript; that there are no conflicts of interest of any kind; that all financial sources are fully and clearly stated in the acknowledgements section; and that they fully agree with the final edited version of the article. A signed document has been filed in the journal archives.

\section{Referencias}

Comisión Nacional del Medio Ambiente (CONAMA). Gestión de Residuos Peligrosos en Chile "Proyecto ResPel". Guía para la Elaboración de Planes de Manejo de Residuos Peligrosos. Proyecto CONAMA/GTZ: Santiago, Chile, 2005.

Escuela de Ciencias Exactas y Naturales (2019). PROLAB: Inicio. Recuperado de https://www.uned.ac.cr/ecen/prolab/inicio

Fondo Social Europeo. Gestión de Residuos Industriales. Guía para la intervención de los trabajadores. Instituto Sindical de Trabajo, Ambiente y Salud: Madrid, España, 2010.

Geocycle. Eco-procesamiento. http://www.eco-procesamiento.com.co/soluciones.html (Accesado el 25 de octubre de 2019).

Grupo Nacional Coordinador. (2008). Perfil nacional sobre la gestión racional de sustancias químicas (Primera edición). Heredia, Costa Rica: EUNA.

Martínez, J. (2005) Guía para la Gestión Integral de Residuos Peligrosos. Fundamentos, tomo I; Centro Coordinador del Convenio de Basilea para América Latina y el Caribe: Montevideo, Uruguay, pp. 11,12.

Ministerio de Salud (2019). Autorizaciones y Certificaciones: Movimiento Transfronterizo de Residuos Peligrosos. Recuperado de https://www.ministeriodesalud.go.cr/index.php/679-tramites/autorizaciones-y-certificaciones/654-autorizaciones-paradesechos-peligrosos

Ministerio de Salud. (2016). Plan Nacional para la Gestión Integral de Residuos 2016-2021. Recuperado de https://www.ministeriodesalud.go.cr/index.php/biblioteca-de-archivos/sobre-el-ministerio/politcas-y-planes-ensalud/planes-en-salud/3025-plan-nacional-para-la-gestion-integral-de-residuos-2016-2021/file

Mora, J. (2010). Generación de un Diagnóstico en la Gestión Actual de Desechos Químicos en la Universidad Nacional. (tesis de maestría). Universidad Nacional, Heredia, Costa Rica.

Poder Ejecutivo. (29 de abril de 1998). Decreto N²7000-MINAE: Reglamento sobre las características y listado de los desechos peligrosos industriales. La Gaceta.

Poder Ejecutivo. (29 de abril de 1998). Decreto N 27001-MINAE: Manejo de Desechos Peligrosos Industriales. La Gaceta.

Ramírez, K. (16 de abril de 2018) . PROLAB cumple 40 años con modernos equipos para las ciencias. Acontecer Digital. Recuperado de https://www.uned.ac.cr/acontecer/a-diario/juncos/51-a-diario-/gestion-universitaria/3162-prolab-cumple40-anos-con-modernos-equipos-para-las-ciencias

Universidad Estatal a Distancia (2012). Plan de Mejoramiento Institucional. Iniciativas financiadas con los fondos acordados en el Artículo 12 del V Convenio de Negociación del FEES. Recuperado de https://www.uned.ac.cr/ami/iniciativas

Vallero, D. A. (2019). Chapter 31 - Hazardous Wastes. Waste, 585-630. https://doi.org/10.1016/B978-0-12-815060-3.00031-1

Vargas-Camareno, M., \& Romero-Esquivel, L. (2006). Tecnología de inmovilización de desechos peligrosos en Costa Rica. Revista Tecnología En Marcha, 19(3), pág. 3-8. Recuperado a partir de https://revistas.tec.ac.cr/index.php/tec_marcha/article/view/2 